\title{
Reading the Light Right: The Exposure of Asian Skin Tones in Cinematography
}

\author{
Yu-Lun Sung
}

https://doi.org/10.15664/fcj.v0i17.2075

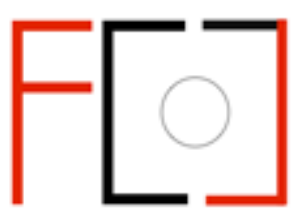

Frames Cinema Journal

ISSN 2053-8812

Issue 17 (Jun 2020)

(c) $\underset{\mathrm{Er}}{(-)}$

http://www.framescinemajournal.com 


\section{Reading the Light Right: The Exposure of Asian Skin Tones in Cinematography Yu-Lun Sung}

This article discusses how contemporary cinematographers make technical and aesthetic decisions about exposure when working with actors of different skin colours - specifically those of Asian ethnicities. Having worked internationally as a cinematographer and lighting co-ordinator, with casts belonging to different ethnicity groups, I am able to offer practical insight into the interaction between colour, the quality of light, and Asian skin in digital film production. As I will explore below, it is encouraging to see that cinematographers have begun exploring ways to better illuminate Black actors, and arguably, the same level of care and creativity can be applied when filming Asian talent. The article will examine firstly, how exposure tools for the camera are used, and secondly, the different approaches of industry practitioners seeking to develop more careful and varied ways of lighting Asian skin.

Lorna Roth, who has written extensively on-screen diversity, Indigenous media representations, and colour media, argues that in order to understand racial equality issues, we need to address questions of 'cognitive equality.' She proposes an intelligent strategy for fostering equity by inscribing a wider range of skin tones into imagery technologies, products, and emergent audiovisual practices. ${ }^{1}$ She suggests we must be more open to the possibilities of technologies to enable racial inclusiveness, and I suggest that this can be applied to cinematographers, who must recognise and investigate the range and subtleties of Asian skin colours by normalising them. ${ }^{2}$ In this article, I attempt to develop a way of looking at film lighting which takes account of the interaction between light and the physiological attributes of Asian skin, and how this impacts cultural perceptions of skin colour. My focus will be on three case studies, Columbus (Kogonada, 2017), Crasy Rich Asians (Chu, 2018), and The Farewell (Wang, 2019), in which the cinematographers adopt different strategies to represent the unique characteristics and qualities of a range of Asian skin tones.

Certain areas are outside the scope of this study, such as make-up, colour grading, and film stock specifications. For instance, John Akomfrah states that re-negotiations of realism in Black representation have led to debates over the "inherent 'biases' of film stock," arguing that the "film processing laboratories, set up to process these stocks, worked with a 'correct exposure truth' which increasingly worked against appropriate black skin tones." ${ }^{\prime 3}$ Make-up and grading are also closely associated with lighting, but there is not the space to consider them in in depth here. ${ }^{4}$ It should also be acknowledged that the case studies I discuss were produced in a US production context, and to a significant extent are aimed at western audiences. My aim here is to explore some more general, practical techniques developed by cinematographers in lighting and representing Asian skin tones. This will lay the groundwork for future research, which can take full account of other factors that impact on lighting (make-up, film stock, colour grading), diversity in regional practice, and films intended primarily for Asian audiences.

\section{Calibrating Whiteness in Lighting}

The manipulation of light is one of the most crucial skills in the cinematographer's trade. Light interacts with celluloid or photo-sites built into a sensor, creating the images for storytelling, 
regardless of whether the reproductions result from a photochemical reaction or are digitally coded. Striving to master their craft, cinematographers study the light surrounding them and resolve to make judgements on exposure - the numerical result of light reading that corresponds to the lens aperture - in order to control the light passing through, and to achieve the desired images.

Practitioners such as Blain Brown and Paul Wheeler have respectively in their books Motion Picture and Video Lighting ([1992] 2008) and Practical Cinematography ([2000] 2005), discussed the importance of exposure when constructing images, explaining how cinematographers are trained to utilise light meters for accuracy. ${ }^{5}$ All types of light meters operate on the premise that they are assessing something that has a reflectance value of 18 per cent (reflectance value here refers to how much light is reflected from the surface on which the light lands). In verifying this value, cinematographers set the camera lens to match 18 per cent, which is the average reflectance in everyday settings. This corresponds to the photographed image being in Zone $\mathrm{V}$ of the Zone system formulated by photographer Ansel Adams. ${ }^{6}$ Applied in cinematography, this helps create a scene with brightness differentiation, the difference between the darkest (blackest) and the brightest (whitest) parts of an image, facilitating the process of exposure control (see Figure 1 \& $2)^{7}$

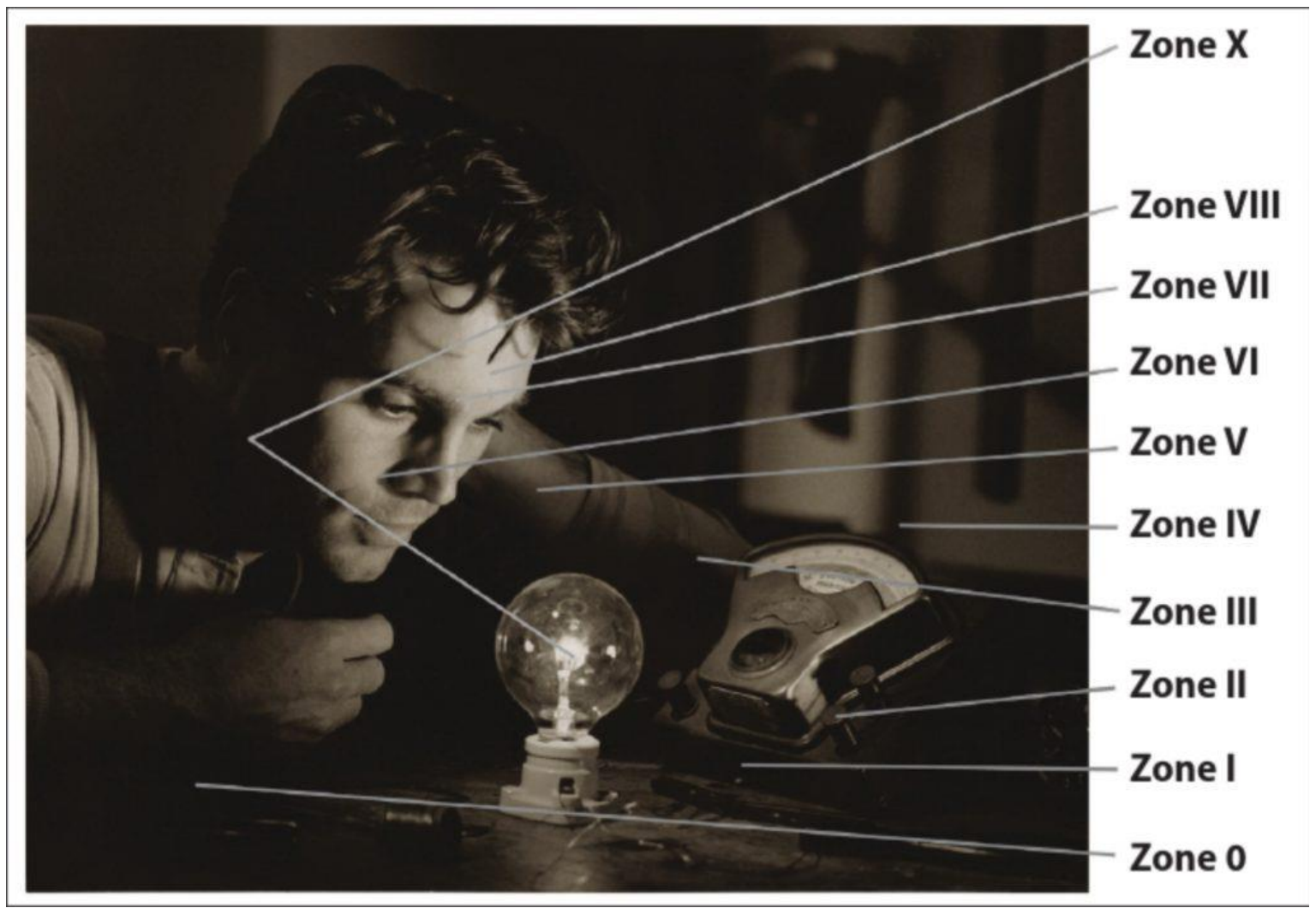

Figure 1: Zones in a black-and-white print 1 (Blain Brown, Cinematography, 204) 


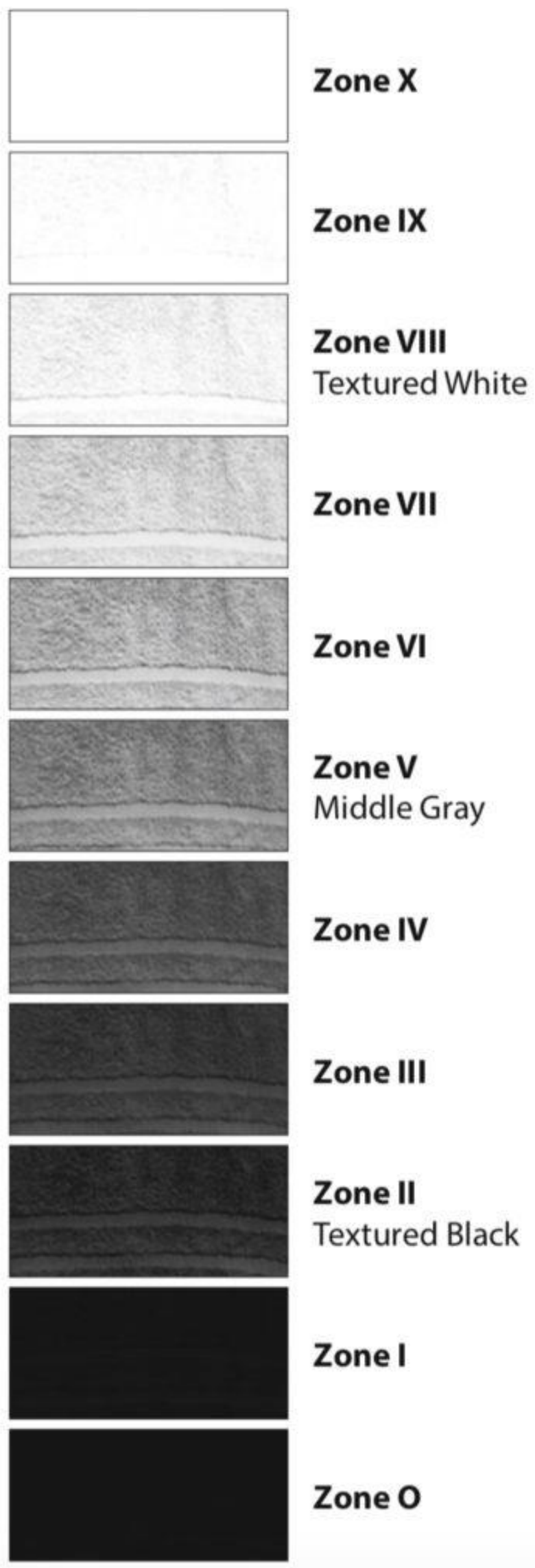

Figure 2: Zones as shown by photographing a towel in sunlight, illustrating texture and detail (Blain Brown, The Filmmaker's Guide to Digital Imaging for Cinematographers, 119) 
Typically, when learning exposure as discussed above, Caucasian skin is taken to be the reference point. $^{8}$ The reflectance of Caucasian skin sits in Zone VI, and the texts by Brown and Wheeler conclude that this is one of the few constants cinematographers can count on. If you take the reading of Caucasian skin with your light meter, you can confidently open up the aperture of your lens by one stop, in order to expose your images in Zone $\mathrm{V}$, a reasonable working average of brightness when photographing a typical scene.

This long-established convention raises questions when academics and practitioners seek to investigate or depict non-white skin tones. John Alton, in his book Painting with Light (1949), wrote that filmmakers had developed work-around methods to decide the exposure for darker-skinned actors. 'When shooting In the Heat of the Night (Jewison, 1967), Director of Photography (DP) Haskell Wexler toned down the light on lead actor Sidney Poitier for a better response to his skin complexion, rather than adding more light which might have been a more intuitive approach. ${ }^{10}$ In another canonical how-to manual, the Set Lighting Technician's Handbook (1993), Harry C. Box advises readers to accommodate different skin attributes when making necessary compensations in light reading. ${ }^{11}$ However, a consideration of Black and Asian skin is often an afterthought, offering complimentary knowledge appended to a baseline norm - Caucasian, white skin. Richard Dyer discusses the cultural mechanisms that have formed and reproduced this white hegemony in Western visual culture. He argues that "the photographic media and, a fortiori, movie lighting assume, privilege and construct whiteness. The apparatus was developed with white people in mind and habitual use and instruction continue in the same vein." ${ }^{\prime 2}$ While some practitioners have begun to consider how to challenge this, the representation of non-white skin tones as the first and foremost consideration during shooting and post-production has not been sufficiently discussed. No systematic method has been developed that optimises the filming of Asian skin, whereas there are some examples of filmmakers addressing this in relation to Black subjects. ${ }^{13}$

\section{Visualising Different Skin Tones}

Asian skin, like Caucasian and African skin, is composed of three parts: the epidermis, dermis, and hypodermis, but it has a larger amount of melanin in the epidermis region compared to the Caucasian equivalent. The amount of melanin produced is genetically predisposed, and is the primary factor deciding the shade of people's skin. Furthermore, the luminance of skin is mainly determined by the amount of melanin, as heavily pigmented skin absorbs more light. There are two types of melanin contributing to an individual's subtler skin colour differences: red/yellow pheomelanin and brown/black eumelanin. ${ }^{14}$ All skin tones have unique physiological combinations which create nuanced colours, and care is needed in representing them.

In order to create flattering representations of skin, cinematographers need to contemplate its reflectance, its unique colour pigmentation, and the preferred skin tone of the audience, which is culturally specific. ${ }^{15}$ Studies suggest, for example, that preferred skin representation may vary in different Asian regions. ${ }^{16}$ Huanzhao Zeng, for example, concludes that Chinese subjects prefer a slightly less chromatic Asian skin colour, whereas Kok Wei Tan suggests Malaysian Chinese prefer yellower, but less red skin. ${ }^{17}$ Discussing colour correction, Alexis Van Hurkman demonstrates that physiological characteristics play an important role in the interaction between light and skin, and 
result in different outcomes when filming occurs. Too much yellow or green could result in the photographed subject being seen as unnaturally sick, while too much red might imply sunburn or embarrassment. ${ }^{18}$

Having explained the biological characteristics of skin, I will now discuss visual work featuring Black subjects that offers some practical solutions when filming darker skin tones. DP Cybel Martin shares her insights of having worked with dark brown skin, that tends to possess blue undertones; similar to the colour correction procedure when working with different colour temperature lights, she suggests that by adding a $1 / 4 \mathrm{CTO}$ (colour temperature orange) gel to the lights, the blue wave reflected from the skin can be neutralised. To preserve the ebony colour of darker-skinned actors, she also suggests not flooding their faces with an excess of light, and to instead use a side 'kicker' to outline the shape of their face and bone structure, to differentiate their figures from the background. ${ }^{19}$ Furthermore she also recommends using a soft, bounced single source for darker-skinned cast, which allows the light to wrap around the subject's skin from right angles, and avoids leaving a 'hot spot' (unpleasant overexposed area) on the actor's face. ${ }^{20}$

Contextualising the abovementioned techniques, I will now analyse my three case studies, in order to inform some hypothetical approaches to lighting Asian skin that could be used in the future. I will explain how the cinematographers of these films move beyond the Zone system when approaching Asian skin, and how Asian skin complexions respond to the light which the cinematographers design and project.

As Hollywood begins to tell more stories featuring Asians, viewers should be allowed to see the varieties and subtleties of their skin, and in this respect a study of Asian skin in cinematography seems timely. Crasy Rich Asians was the first Hollywood romantic comedy to feature an all-Asian cast since The Joy Luck Club (Wang, 1993), and its release has galvanised conversations around diversity in casting. ${ }^{21}$ Needless to say, its images are produced with high production values, giving the film a blockbuster quality. The Farewell is a bittersweet, light-hearted, but also profound allAsian family drama. Unlike Crayy Rich Asians, it has a more realistic tone, with its modest lighting approach seeking to emphasise the authenticity of bringing two cultures into collision. Finally, Columbus, about a young American woman who encounters a distant, mature Korean man, heavily utilises 'practical' lights (lights that already exist in the location, or can be added to look like they are in situ) and natural light. The lighting interacts with the space, to bring out a vivid, saturated colour palette.

The cast of Crasy Rich Asians are mainly from the Chinese Asian group, with some exceptions, such as the lead actor Henry Golding, who has Malaysian heritage. The cast of The Farewell are also mainly Chinese Asian, with two exceptions, the lead actress Awkwafina (who also appears in Crayy Rich Asians), whose father is Chinese-American and mother is Korean-American, and the supporting actor who plays Aiko, Aoi Mizuhara, who is Japanese. In Columbus, the lead actor John Cho is Asian-American, born in South Korea. These nuances in ethnic background demonstrate the need for future regional studies in a broader context.

1. Bold and prominent edge lights. 
When lighting actors, cinematographers usually deploy three-point lighting, in which the actors are separated from the background while a key light defines the tonality of a scene. In Crayy Rich Asians, when Rachel Chu (Constance Wu) sits in a wedding (Figure 3) and locks her emotional gaze on her off-frame lover, DP Vanja Cernjul adds an edge light to outline her contours more. Rachel's face is not overly lit; on the contrary, her presence has significant differentiation from the crowd, which is achieved by a strong 'kicker' edge light. The warmer edge lights not only accentuate her facial and bodily outlines, but also paint a subtle golden tint, making her smile vividly glint in a cooler, daylight set-up.

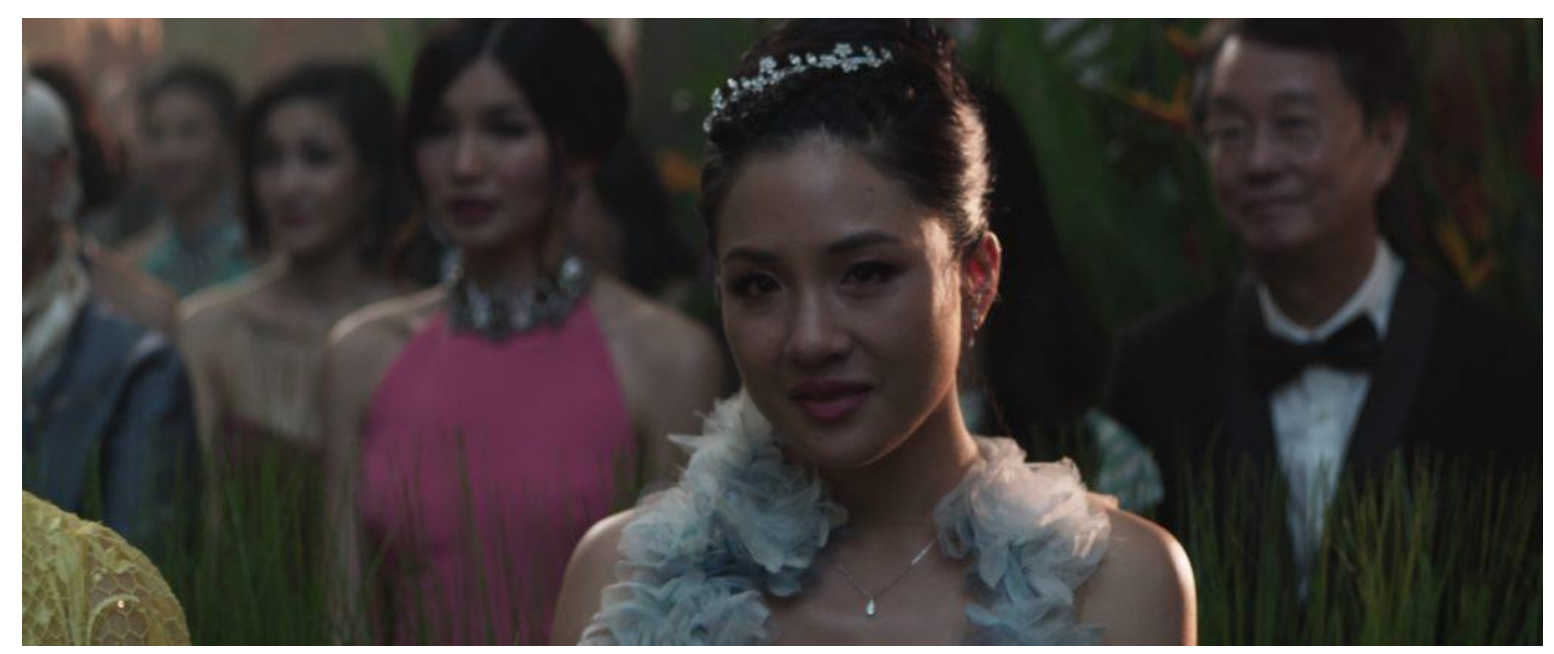

Figure 3: Crasy Rich Asians (Chu, 2018)

Columbus features an intimate frame depicting Casey (Haley Lu Richardson) as she gradually discloses to Jin (John Cho) her past and her love for a building (Figure 4). This demonstrates the aesthetic function of using a 'kicker' edge light on an Asian cast member. Compared to Casey's face, Jin's darker skin tone isn't equally lit like his white female counterpart. DP Elisha Christian projects a cold and harsh blue light, outlining one side of Jin's face. This effectively draws the audience's attention to his performance, sketching out the mystery and callousness of the character's personality. Arguably, these two scenes are crucially important in terms of each film's narrative, and the filmmakers could have lit their lead Asian actors brighter with key light in order that the audience could see them clearly. Dyer elaborates how the premise of the primary function of lighting - to control visibility for guiding the audience to see clearly "what is important in a shot" - explicitly and implicitly constructs and privileges whiteness through photographic apparatus and lighting culture. ${ }^{22}$ Evidently lighting here isn't designed for an equal visibility of each performer; on the contrary, the DPs have chosen to keep the Asian skin tone sitting in a darker zone, and have utilised edge lights to make the Asian actors' faces stand out, which matches the focus of the narratives at these moments. 


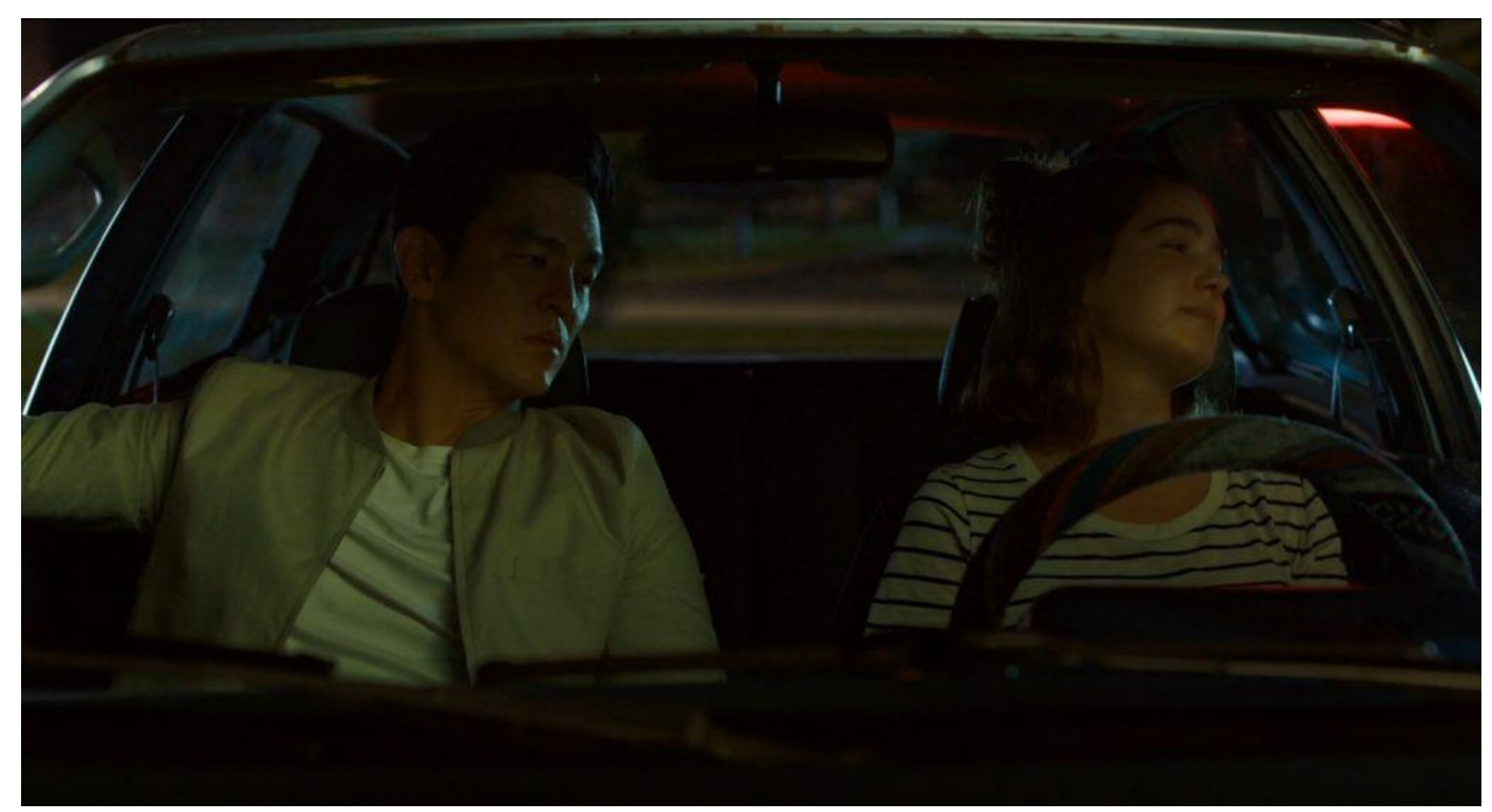

Figure 4: Columbus (Kogonada, 2017)

2. Bounced soft key light source, that creates natural shadow fall-off.

'Rembrandt lighting' is a style that creates a conspicuous triangle highlight on the shadow-side cheek. It is commonly used with men to mould the shape of their face, to emphasises its texture, and to make the scene more dramatic and dimensional. ${ }^{23}$ This one-source lighting can be easily paired with other light modifiers, a soft-box set-up, which gives the DP greater control in achieving the desired light-shadow ratio. DP Bradford Young claims that working with a Black cast, one can "give the skin an opportunity to reflect the environment" with bounce light. ${ }^{24}$ In The Farewell, there is a heart-wrenching moment when Billi (Awkwafina) struggles to conceal the secret of her beloved Nai-Nai's (Shuzhen Zhao) cancer diagnosis (Figure 5). She has been weighing her western morals against eastern family values, and her internal wrestling is almost reaching breaking point, as NaiNai, oblivious to her illness, expresses her care and love to Billi. In moulding the actress's face with bounced light, creating a smooth shadow fall-off from the left side of Billi's face (her skin colour accentuates the shades from bright to dark), DP Anna Franquesa Solana deploys a single, soft source to successfully portray Awkwafina's subtle expression elegantly. 


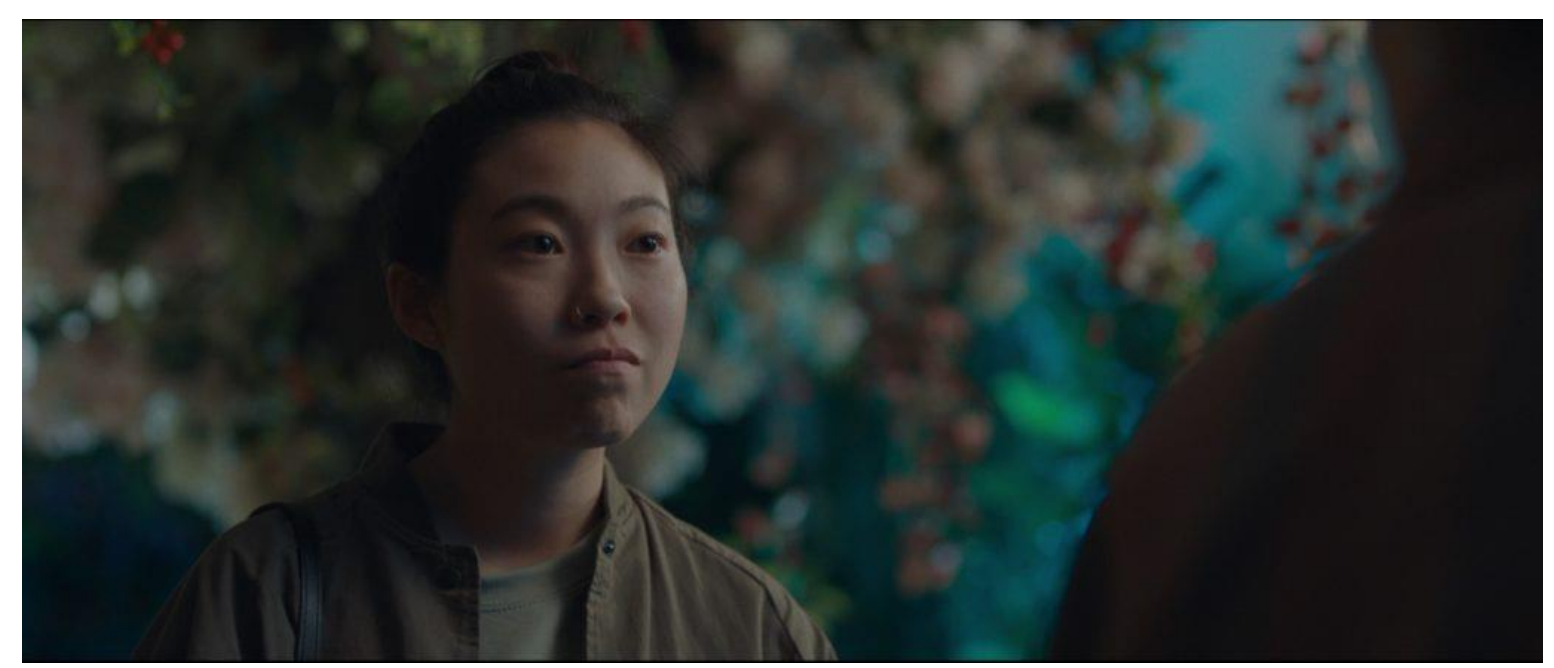

Figure 5: The Farewell (Wang, 2019)

A similar effect can be found in an example from Columbus (Figure 6), in which a golden soft light, from the bottom-left side of the frame, gently illuminates Jin's face. He is treading carefully as his interlocutor Casey hesitates in revealing her mother's substance addiction. This frame is the first time we see Jin in close-up during this scene, and his face is lit with gleaming incandescent light. The beauty of this frame comes from his olive skin bouncing the golden aura, and the audience can feel, as if sharing Casey's gaze, Jin's warm tenderness.

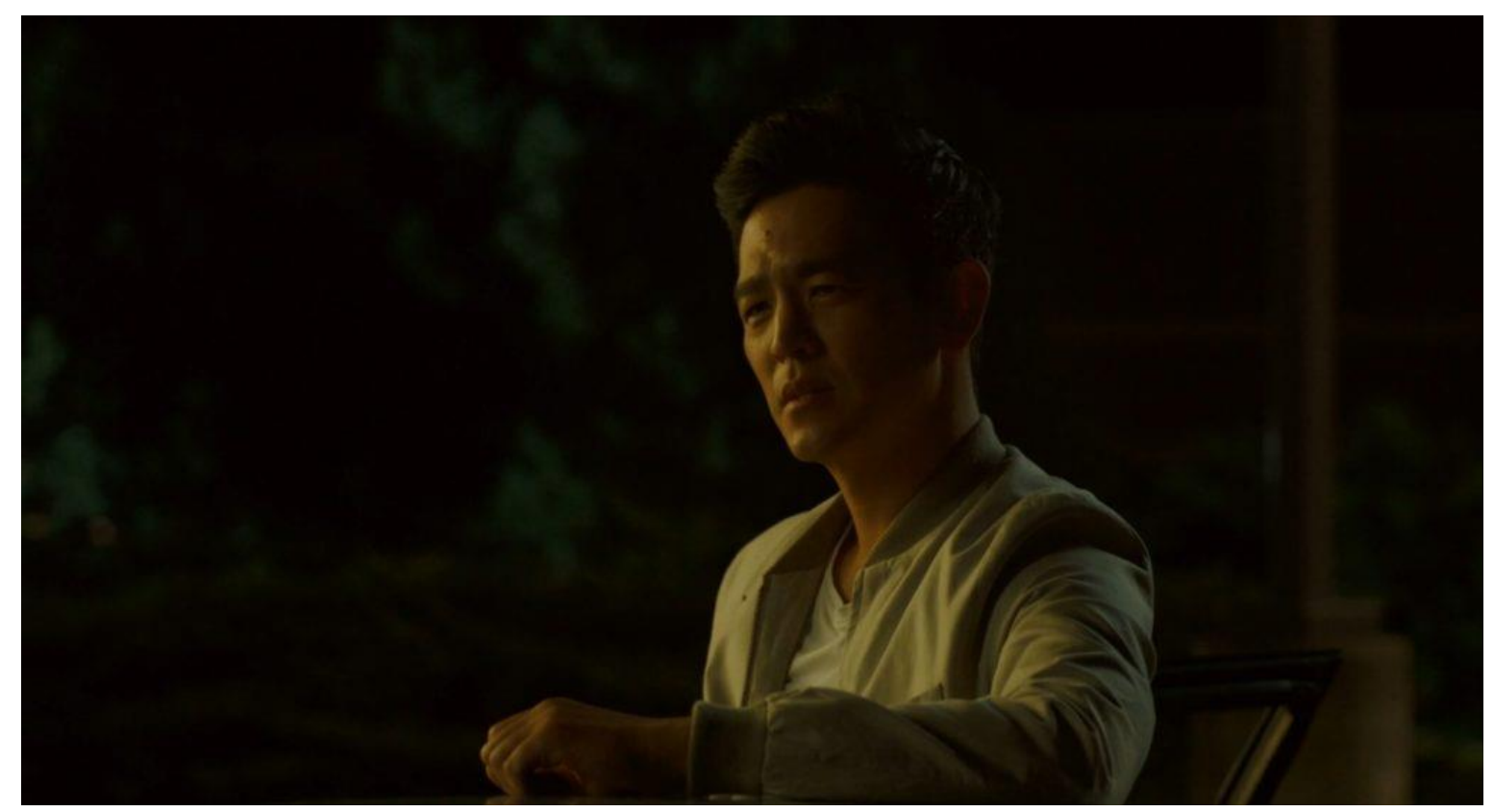

Figure 6: Columbus (Kogonada, 2017)

3. Pink-magenta bounce boards as a fine light brush.

In Marina Starke's thesis, The Visual Appearance of Skin in Motion Picture (2017), she cites her interview with industry colourist Kevin Shaw from Colourist Society International (CSI), that offers insights into skin tone preferences amongst different ethnicity groups. When Shaw worked with Asian clients, they loved Asian skin to be given a vibrant, pink-magenta, baby-like skin which 
leans towards white. ${ }^{25}$ Certainly, magenta is not the ideal colour when cinematographers consider colour schemes, as it is usually an unwanted colour contamination when trying to correct white balance. However, in a scene from The Farewell (Figure 7), a magenta tonality permeates the midtones. The faces of Aiko and Nai Nai shine a cherry blossom luminance; this could result from the effect of colour grading, or the decorations (added as set dressing) reflecting the magenta light. Similarly, in a scene from Crazy Rich Asians (Figure 8), the prominent colours in the set dressing and costumes - magenta and red - serve as bounce sources for neutral white light, which give this scene a pleasing and harmonious look. The skin of Rachel and Peik Lin Goh (Awkwafina) are presented with angelic pink undertones.

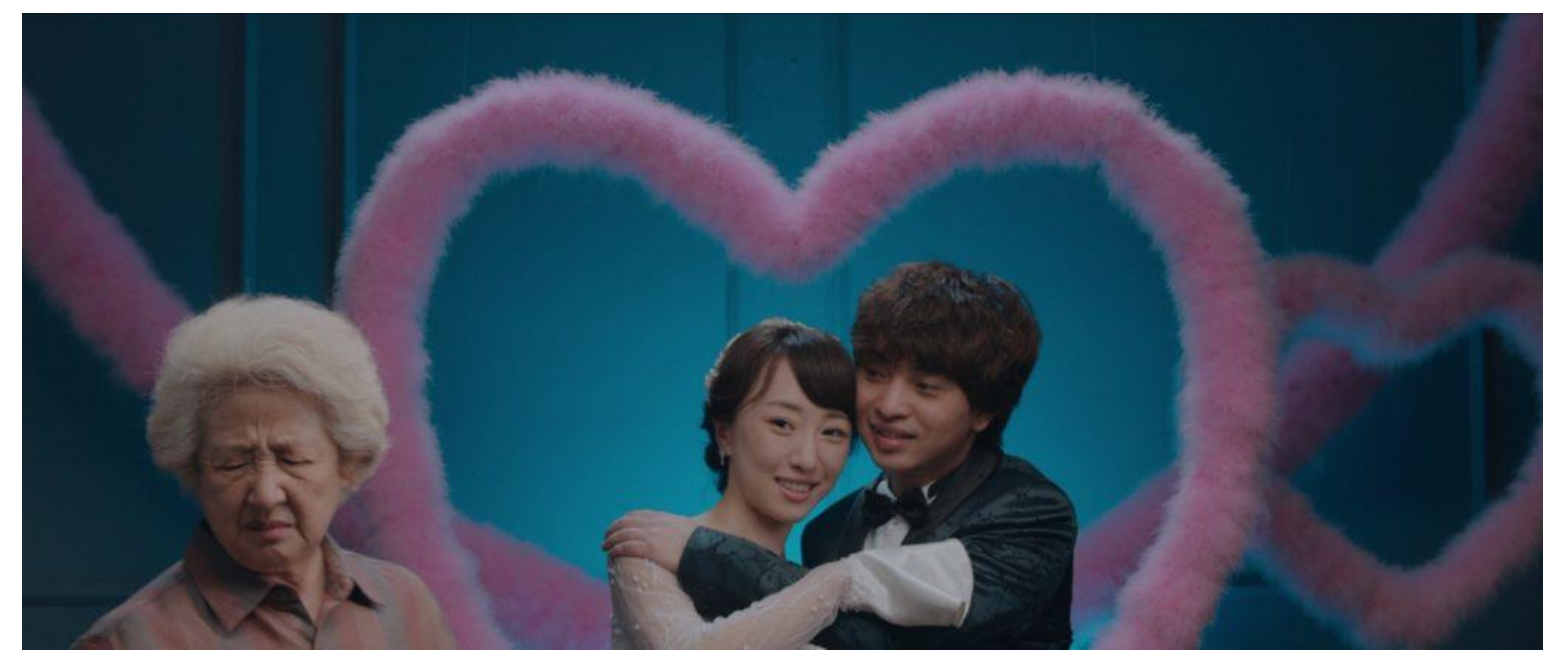

Figure 7: The Farewell (Wang, 2019)

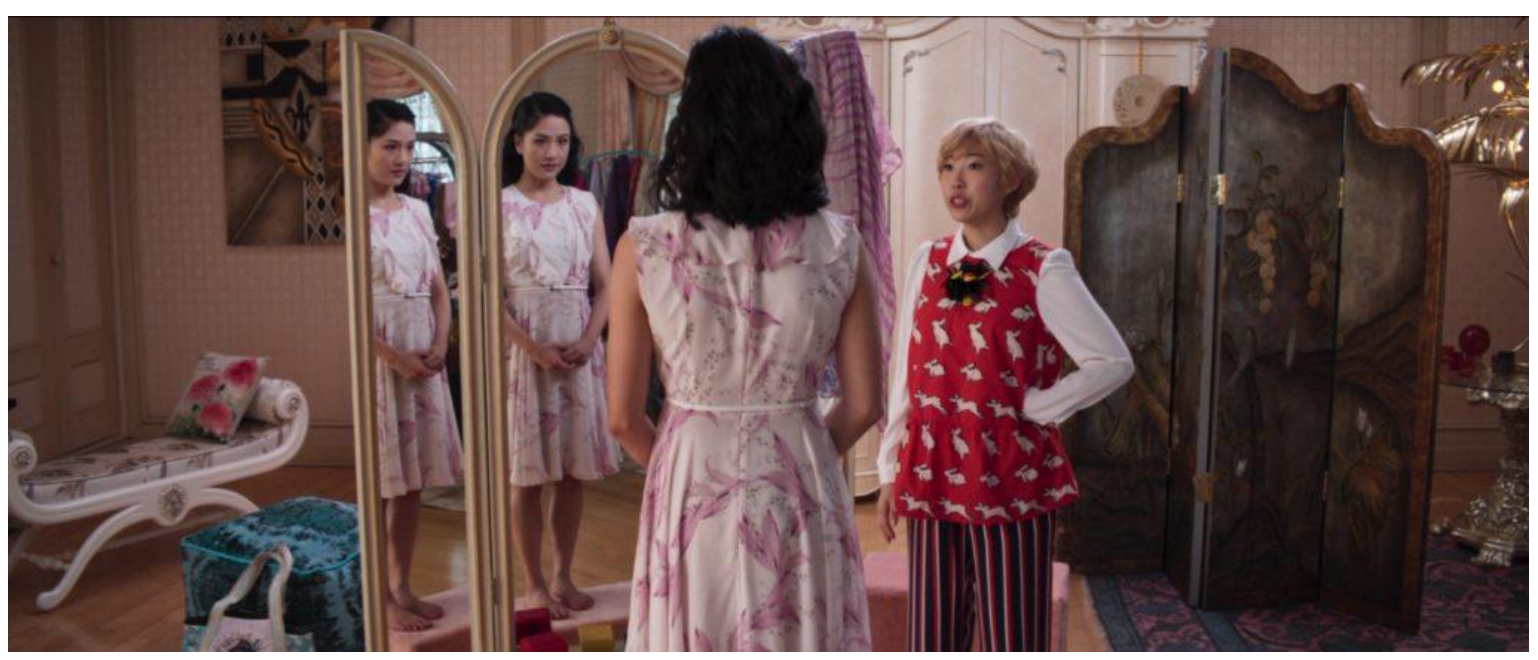

Figure 8: Crasy Rich Asians (Chu, 2018)

As a cinematographer, my experience in fine-tuning adjustments to colour often involves the application of colour bounce cards, whereby the skin will have the desired colours added, while retaining, by and large, the colour temperature coming from the light itself. In so doing, cinematographers can use bounced light to brush the skin with a certain tincture, leaving an uncontaminated image for future colour grading. 
The delicate interaction between Asian skin and lighting requires further research. There are no established aesthetic guidelines yet, and studies suggest the skin preferences of different ethnicity groups may be culturally specific. Further technical questions need to be asked, such as: what type of colour gels and light modifiers can work effectively to achieve the desired results with Asian actors? Where does the balance lie when using a light meter or digital monitor to judge the average brightness, when a multiracial cast appears in the same frame? What digital post-production workflows can privilege a broad range of Asian skin qualities? Cinematographers are proud of their expertise in using lighting to make a subject look 'right' for the needs of the narrative. It is now time to develop a new system to effectively guide us in better understanding and representing Asian skin in all sorts of light.

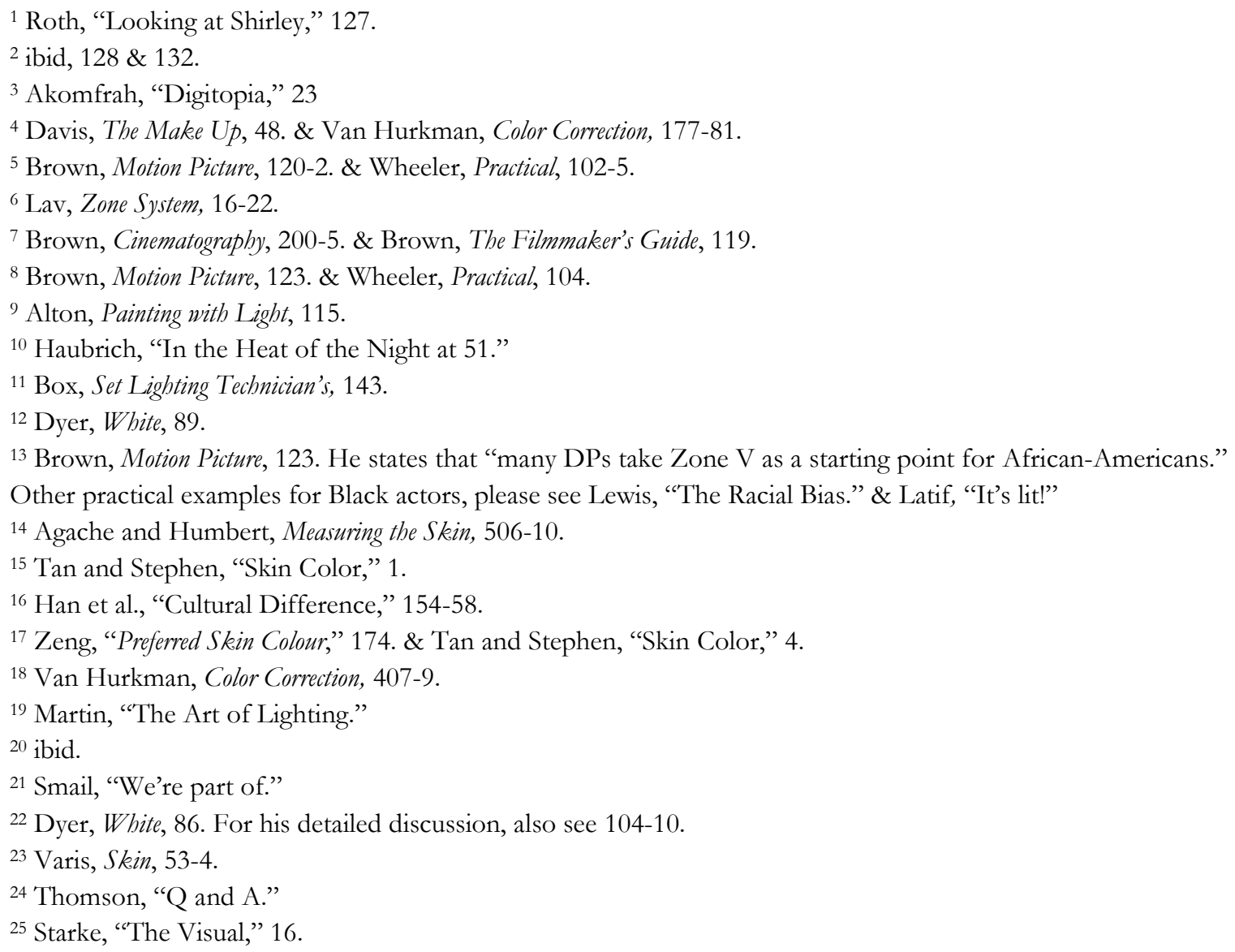




\section{Bibliography}

Agache, Pierre, and Philippe Humbert, Measuring the Skin. Berlin: Springer, 2011.

Akomfrah, John. "Digitopia and the spectres of diaspora." Journal of Media Practice vol. 11, no. 1

(January 2014): 21-29. https://doi.org/10.1386/jmpr.11.1.21/1.

Alton, John. Painting with Light. New York: University of California Press, 1995.

Box, Harry C., Set Lighting Technician's Handbook. Burlington: Elsevier Science, 2003.

Brown, Blain. Cinematography: Theory and Practice. Burlington: Routledge, 2016.

Brown, Blain. Motion Picture and Video Lighting. Burlington: Focal Press, 2008.

Brown, Blain. The Filmmaker's Guide to Digital Imaging: for Cinematographers. Burlington: Focal Press, 2015.

Davis, Gretchen, and Mindy Hall. The Makeup Artist Handbook. Burlington: Focal Press, 2012.

Dyer, Richard. White. New York: Routledge, 2017.

Han, Chengyang, Hongyi Wang, Amanda C. Hahn, Claire I. Fisher, Michal Kandrik, Vanessa

Fasolt, Danielle K. Morrison et al.. "Cultural differences in preferences for facial coloration." Evolution and Human Behavior 39(2) (December 2017): 154-159. https://doi: 10.1016/j.evolhumbehav.2017.11.005.

Haubrich, Wess. "In the Heat of the Night at 51: still incredibly innovative." Medium, June 4, 2018. https://medium.com/@HaubrichNoir/depicting-african-american-actors-on-filmwhy-in-the-heat-of-the-night-remains-insanely-innovative-5bb2d5608aea.

Latif, Nadia. "It's lit! How film finally learned to light black skin." The Guardian, September 21, 2017. https://www.theguardian.com/film/2017/sep/21/its-lit-how-film-finally-learnedhow-to-light-black-skin.

Lav, Brian. Zone System: Step-by-Step Guide for Photographers. Buffalo: Amherst Media, Inc., 2002.

Lewis, Sarah. "The Racial Bias Built into Photography." The New York Times, April 25, 2019. https://www.nytimes.com/2019/04/25/lens/sarah-lewis-racial-bias-photography.html.

Martin, Cybel. "The Art of Lighting Dark Skin for Film and HD," Shadow and Act, April 20, 2017. https://shadowandact.com/2014/02/04/the-art-of-lighting-dark-skin-for-filmand-hd/.

Roth, Lorna. "Looking at Shirley, the Ultimate Norm: Colour Balance, Image Technologies, and Cognitive Equity." Canadian Journal of Communication, vol. 34, no. 1 (2009): 111-136. https://doi.org/10.22230/cjc.2009v34n1a2196

Smail, Gretchen. “'We're part of a greater movement': Hollywood finally gives Asian stories a spotlight." The Guardian, August 15, 2018. https://www.theguardian.com/film/2018/aug/15/hollywood-asian-stories-crazy-richasians-to-all-the-boys-ive-loved-before-kevin-kwan-jenny-han.

Starke, Marina. “The Visual Appearance of Skin in Motion Picture.” Bachelor Thesis, Media University Stuttgart, 2017.

Tan, Kok Wei, and Ian D. Stephen. "Skin Color Preferences in a Malaysian Chinese Population." Frontiers in Psychology vol. 10 (June 2019): 1-6. https://doi.org/10.3389/fpsyg.2019.01352

Thomson, Patricia. "Q and A with Bradford Young." The American Society of Cinematographers, February Issue, 2015. https://theasc.com/ac_magazine/February2015/QandAwithBradfordYoung/page1.htm 1

Van Hurkman, Alexis. Color Correction Handbook (2 ${ }^{\text {nd }}$ ed.). San Francisco: Peachpit Press, 2014.

Wheeler, Paul. Practical Cinematography. Burlington: Focal Press, 2005. 
Zen, Huanzhao. "Preferred Skin Colour Reproduction.” PhD Diss., University of Leeds, 2011.

\title{
Filmography
}

In the Heat of the Night (Norman Jewison, 1967)

The Joy Luck Club (Wayne Wang, 1993)

Columbus (Kogonada, 2017)

Crazy Rich Asians (Jon Chu, 2018)

The Farewell (Lulu Wang, 2019)

\begin{abstract}
About the Author
Yu-Lun Sung is Lecturer in Cinematography in the School of Arts and Creative Industries at London South Bank University. He works internationally as a cinematographer and coordinator specialising in camera, lighting and grip departments. He worked as an on-set lighting coordinator on Martin Scorsese's Silence, and was the cinematography coordinator for the Chinese Director of Photography Shi Luan. His past works include commercials, music videos and short films, which have been broadcast on TV channels and online. One of many short films Luc has shot as DP, To Pluto, won an award at the British Independent Film Festival, and Best Picture at the 2016 MOD Golden Short Film Competition. His research interests include representations of race in cinematography and film noir, contemporary French female directors of photography, and cultural differences in international co-production.
\end{abstract}

